\title{
La rurbanisation du versant vosgien du Parc Naturel Régional des Ballons des Vosges et l'exemple du village de Provenchères-sur-Fave
}

Rurbanisation of the Vosgian side in the natural parc of Ballons des Vosges and the example of the village Provenchères-sur-Fave

Die Stadtumlandwanderung im Naturpark der Ballons der Vogesen. Das Beispiel des Vogesenhanges und des Dorfes Provenchères-sur-Faye

\section{Vincent Bertrand}

\section{OpenEdition}

Journals

Édition électronique

URL : http://journals.openedition.org/rge/2377

ISSN : 2108-6478

Éditeur

Association des géographes de l'Est

Édition imprimée

Date de publication : 1 juin 2003

ISSN : 0035-3213

Référence électronique

Vincent Bertrand, "La rurbanisation du versant vosgien du Parc Naturel Régional des Ballons des Vosges et l'exemple du village de Provenchères-sur-Fave », Revue Géographique de l'Est [En ligne], vol 43 / 3 | 2003, mis en ligne le 26 novembre 2010, consulté le 07 septembre 2020. URL : http:// journals.openedition.org/rge/2377

Ce document a été généré automatiquement le 7 septembre 2020

Tous droits réservés 


\section{La rurbanisation du versant vosgien du Parc Naturel Régional des Ballons des Vosges et l'exemple du village de Provenchères-sur-Fave}

Rurbanisation of the Vosgian side in the natural parc of Ballons des Vosges and the example of the village Provenchères-sur-Fave

Die Stadtumlandwanderung im Naturpark der Ballons der Vogesen. Das Beispiel des Vogesenhanges und des Dorfes Provenchères-sur-Faye

\section{Vincent Bertrand}

\section{NOTE DE L'AUTEUR}

NB : Les données statistiques faisant état des effectifs de population (densités, et variations) reprennent les chiffres du recensement de 1999. Les données portant sur les flux pendulaires datent de 1990 : la politique commerciale de l'INSEE n'ayant pas permis à l'auteur d'actualiser l'ensemble des données.

Le texte reprend une communication faite par l'auteur, le vendredi 19 novembre 1999, lors du séminaire organisé par le Parc Naturel des Ballons des Vosges et intitulé :

"Villes, bourgs, villages-centre..." 


\section{Introduction}

La rurbanisation constitue une forme nouvelle d'organisation de l'espace rural. Elle se caractérise :

- par des flux de personnes (migrations définitives et pendulaires, déplacements liés aux achats et aux loisirs, etc.) et des flux financiers (salaires des migrants pendulaires, dépenses des ruraux en ville, mouvements fonciers, etc.) ;

- par la transformation des villages (constructions pavillonnaires, réhabilitations de vieilles fermes, variations brutales d'effectifs de population, réouvertures de classes ou de commerces et nouveaux rapports entre habitants);

- par une fonction-dortoir qui progressivement concerne l'essentiel des actifs du village.

La rurbanisation résulte d'un choix de vie et plus exactement d'un choix d'espace-vécu motivé par le besoin de calme, de sécurité et de nature incarné par la campagne dans l'esprit de certains, et par le désir d'accéder à la propriété d'une partie importante de la population. Elle est rendue possible depuis les années soixante par l'accroissement du niveau de vie général, par les efforts d'équipement des campagnes et surtout le développement spectaculaire du nombre des véhicules particuliers et de la qualité des réseaux routiers et autoroutiers. Elle est ainsi à l'origine d'un nouveau rapport à l'espace pour une partie croissante de la population et elle se retrouve au cœur des relations villes-campagnes.

L'étude de la rurbanisation impose de multiplier les échelles d'analyse. Les flux pendulaires liés au travail concernent généralement un bassin de vie tout entier qui peut englober plusieurs villes et des dizaines, voire des centaines de villages. Par contre, pour suivre les transformations paysagères ou fonctionnelles liées à la rurbanisation, il importe de descendre à une échelle plus fine, celle du village. Nous nous sommes ici limités au seul versant vosgien du Parc Naturel Régional des Ballons des Vosges (PNRBV) et nous avons retenu une commune : Provenchères-sur-Fave. Cette approche multiscalaire assez originale est dictée par la nature même de la rurbanisation, et elle explique pourquoi depuis trente ans le phénomène demeure étranger aux actions des pouvoirs publics peu préparés à agir à des échelles aussi différentes.

\section{Le versant vosgien du PNRBV : un versant apparemment urbain}

4 Le versant vosgien du Parc est tout en contraste. Des trois versants du Massif Vosgien c'est apparemment le plus urbanisé. Plus des deux tiers de ses habitants résident dans des communes de plus de 2000 habitants (tableau $n^{\circ} 1$ ). De même, une commune sur quatre y dépasse ce seuil contre une sur dix pour les versants franc-comtois et alsacien (tableau $\mathrm{n}^{\circ} 1$ ). Ainsi, la taille moyenne des communes du versant vosgien est la plus importante (tableau n ${ }^{\circ} 1$ ) : 1457 habitants contre respectivement 753 et 1006 habitants. Les vallées souvent larges pénètrent profondément dans le massif, favorisant les implantations humaines: industries, routes et villes s'y concentrent. Par contre les versants raides et les sommets arrondis sont abandonnés de plus en plus aux forêts. La population y est rare et éparse. Les territoires communaux comprennent en général une portion transversale de vallée. Ainsi chaque commune dispose de terroirs variés : 
des prairies occupant les fonds de vallées inondables et les premières pentes qu'elles partagent avec quelques champs, des forêts sur les pentes les plus raides et des pâturages d'été sur les sommets.

Tableau 1 : Quelques indicateurs d'urbanisation du PNRBV et de ses différents versants régionaux.

\begin{tabular}{|c|c|c|c|c|c|}
\hline \multirow[t]{3}{*}{1990} & \multicolumn{5}{|c|}{ Alsace } \\
\hline & \multicolumn{2}{|c|}{ communes } & \multicolumn{2}{|c|}{ hab. } & \multirow{2}{*}{$\begin{array}{c}\text { hab./ } \\
\text { commune }\end{array}$} \\
\hline & effectif & $\%$ & effectif & $\%$ & \\
\hline \multirow{6}{*}{$\begin{array}{l}\text { de - de } 2000 \text { hab. } \\
\text { de + de } 2000 \text { hab. } \\
\text { total }\end{array}$} & 128 & 90 & 76563 & 54 & 598 \\
\hline & 14 & 10 & 66398 & 461 & 4743 \\
\hline & 142 & 100 & 142961 & 100 & 1007 \\
\hline & \multicolumn{5}{|c|}{ Lorraine } \\
\hline & \multicolumn{2}{|c|}{ communes } & \multicolumn{2}{|c|}{ hab. (1990) } & hab./ \\
\hline & effectif & $\%$ & effectif & $\%$ & commune \\
\hline \multirow{6}{*}{$\begin{array}{l}\text { de - de } 2000 \text { hab. } \\
\text { de + de } 2000 \text { hab. } \\
\text { total }\end{array}$} & 35 & 76 & 21652 & 32 & 619 \\
\hline & 11 & 24 & 45378 & 681 & 4125 \\
\hline & 46 & 100 & 67030 & 100 & 1457 \\
\hline & \multicolumn{5}{|c|}{ Franche-Comté } \\
\hline & \multicolumn{2}{|c|}{ communes } & \multicolumn{2}{|c|}{ hab. (1990) } & hab./ \\
\hline & effectif & $\%$ & effectif & $\%$ & commune \\
\hline \multirow{6}{*}{$\begin{array}{l}\text { de - de } 2000 \text { hab. } \\
\text { de + de } 2000 \text { hab. } \\
\text { total }\end{array}$} & 44 & 90 & 21073 & 57 & 479 \\
\hline & 5 & 10 & 15857 & $43^{1}$ & 3171 \\
\hline & 49 & 100 & 36930 & 100 & 754 \\
\hline & \multicolumn{5}{|c|}{ PNRBV } \\
\hline & \multicolumn{2}{|c|}{ communes } & \multicolumn{2}{|c|}{ hab. (1990) } & hab./ \\
\hline & effectif & $\%$ & effectif & $\%$ & commune \\
\hline de - de 2000 hab. & 207 & 87 & 119288 & 48 & 576 \\
\hline de + de 2000 hab. & 30 & 13 & 127633 & 52 & 254 \\
\hline total & 237 & 100 & 246921 & 100 & 1042 \\
\hline
\end{tabular}

1. Ce taux d'urbanisation ne prend en compte dans le calcul de la population urbaine que les populations habitant les communes de plus de 2000 habitants. Les résultats diffèrent ainsi sensiblement de ceux de l'INSEE obtenus à partir des agglomérations multicommunales et présentent des chiffres légèrement inférieurs. La principale raison de ce choix vient du type d'habitat vosgien. Considéré comme dispersé, l'habitat vosgien apparaît comme une succession de hameaux disséminés sur de vastes territoires communaux. Avec le temps, de nouvelles maisons sont construites. Toujours distantes des voisines de quelques mètres à quelques dizaines de mètres, elles tapissent le fond des vallées et concourent à joindre les multiples hameaux sans remettre en cause la faible densité des communes. Ainsi, alors que le paysage demeure largement rural d'aspect, la dissémination de l'habitat amène, selon les techniques de calcul de l'INSEE, à un gonflement des taux d'urbanisation.

5 Le versant vosgien du Parc offre aussi une nette opposition entre sa partie nord, davantage rurale, et sa partie sud plus industrielle et urbaine. Au nord, dans le bassinversant de la Meurthe, les communes ont une superficie souvent inférieure ou égale à la moyenne nationale $\left(1529 \mathrm{~km}^{2}\right)$. Par contre, celles du sud dépassent en général 1500 à 2000 hectares, voire atteignent 4 à 5000 pour les plus importantes (Gérardmer, La Bresse ou Cornimont). Ces grandes dimensions permettent à de nombreuses communes d'atteindre le seuil de 2000 habitants, sans pour autant présenter des densités de type urbain (carte $\mathrm{n}^{\circ} 1$ ). Ici se pose un réel problème de définition. Les communes de la partie sud du versant vosgien du Parc offrent, par leur faible densité, un environnement et des paysages plutôt ruraux. Par contre leur poids démographique leur permet de disposer d'équipements dignes d'espaces urbains. Aussi sommes nous face à un environnement original, où les schémas traditionnels opposant villes et campagnes 
sont inadaptés. C'est pourquoi nous avons retenu l'exemple de Provenchères-sur-Fave, chef-lieu d'un canton rural de la partie nord du versant vosgien du Parc.

Carte 1 : Les densités de population du versant vosgien du Parc et de son piedmont.

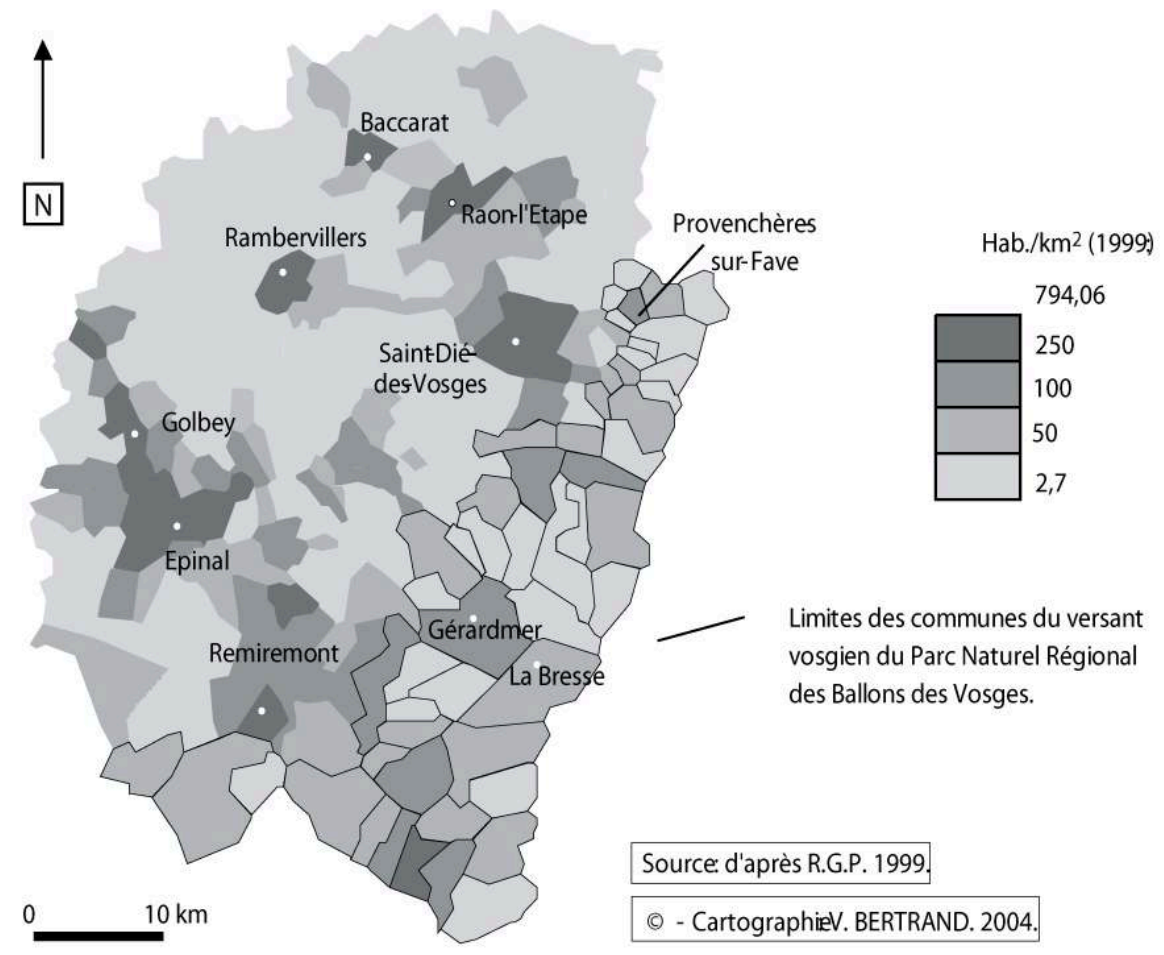

6 Cette opposition entre le nord et le sud de ce versant du Parc se retrouve également au travers des évolutions démographiques. Sur la carte $n^{\circ} 2$, on observe un accroissement relatif de la population des communes rurales ceinturant les principales villes vosgiennes, Epinal et Saint-Dié-des-Vosges. Ainsi, la majorité des communes rurales du versant vosgien du Parc, situées au nord de Gérardmer et La Bresse, gagnent des habitants de 1975 à 1999. Dans le même temps les communes plus vastes, souvent urbaines et situées au sud de ces deux villes, en perdent. Pour mieux comprendre cette situation, l'observation des migrations alternantes se révèle particulièrement intéressante. 
Carte 2 : Un renouveau rural qui profite inégalement aux communes vosgiennes du Parc.

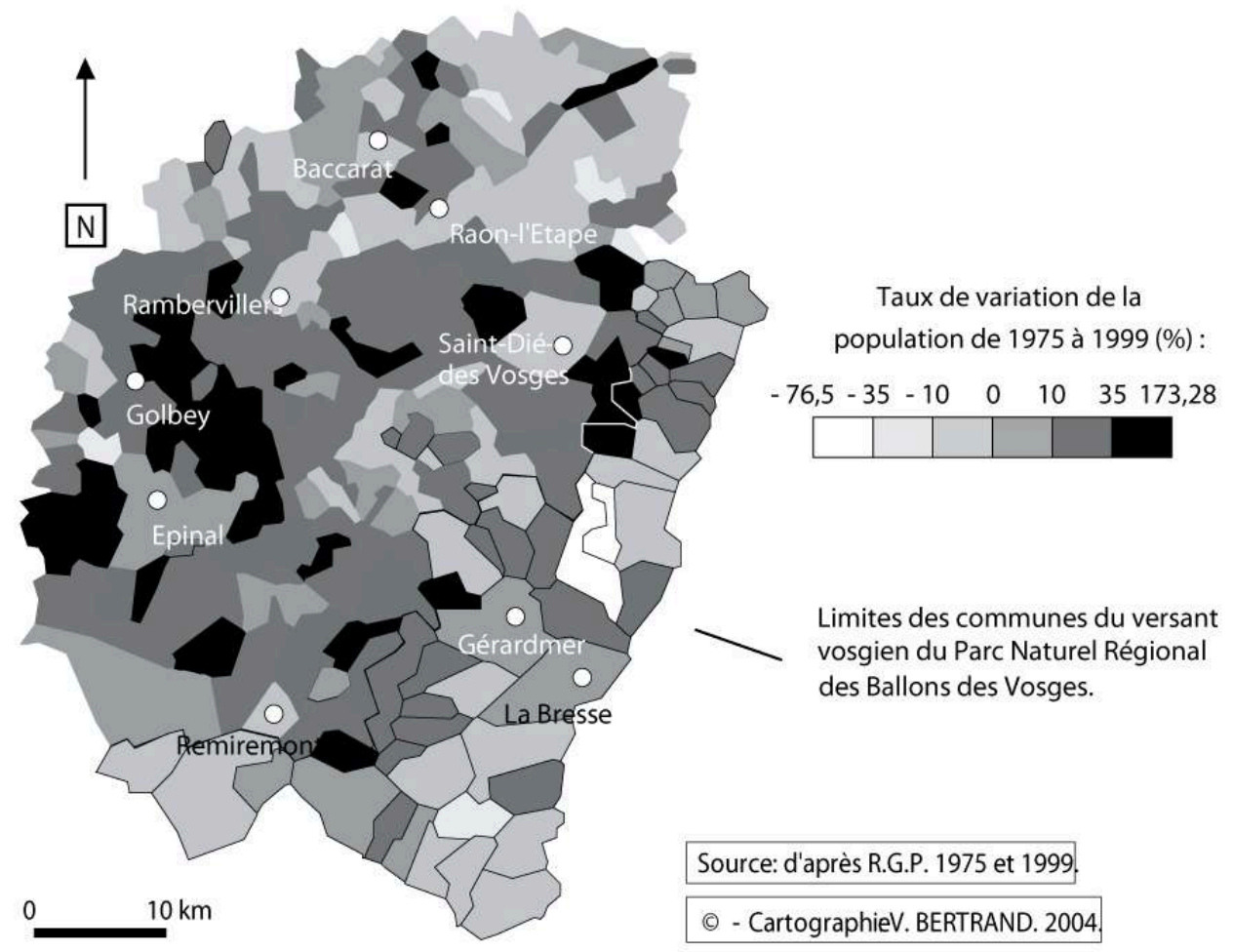

\section{Les migrations pendulaires : un aspect essentiel de la rurbanisation}

7 L'étude des migrations pendulaires impose une échelle d'analyse englobant un à plusieurs bassin(s) de vie, c'est-à-dire un espace présentant une relative cohésion fondée sur une complémentarité des sous-espaces qui le composent. Ces sous-espaces ont des fonctions distinctes: dortoirs, productives (industrielles ou agricoles), commerciales, logistiques ou encore touristiques. Les individus sont ainsi amenés à se rendre plus ou moins régulièrement dans l'un ou l'autre de ces sous-espaces pour satisfaire leurs divers besoins. Leurs déplacements constituent ainsi un des révélateurs de la cohésion d'un territoire.

Pour étudier les migrations alternantes, deux approches sont envisageables. L'une part de la ville et consiste à observer les flux pendulaires qui unissent la campagne à la ville. On observe alors l'attractivité de la ville. Inversement, l'autre consiste à étudier pour chaque village le rapport entre les actifs travaillant en ville, c'est-à-dire les sortants vers la ville, et ceux travaillant à la campagne, autrement dit les stables et les sortants vers la campagne ${ }^{1}$. C'est alors le fonctionnement même du village qui se trouve au cœur des préoccupations. Il n'est plus question d'influence ou d'attractivité urbaine, mais de mode de fonctionnement d'espaces ruraux. L'indicateur clé de ce type d'approche est alors le taux d'actifs travaillant en ville.

9 La carte $n^{\circ} 3$ confirme notre première remarque concernant la définition de la ville dans ce secteur vosgien. En effet, la quasi totalité des communes de la partie sud de ce versant du Parc est considérée comme urbaine par l'INSEE. De fait, l'approche de la rurbanisation à travers le taux d'actifs travaillant en ville devient délicate. Elle reste 
possible dans le seul secteur de la Fave, dans le nord du Parc, et sur le Plateau Lorrain. Le dessin d'auréoles plus ou moins nettes autour des villes principales, Épinal et SaintDié-des-Vosges, montre l'essor plus ou moins important de la rurbanisation dans les communes rurales du Plateau Lorrain. Quant aux communes les plus septentrionales de cette partie du Parc, elles sont inégalement concernées par la rurbanisation. La plupart comptent entre un tiers et la moitié d'actifs travaillant en ville. Une poignée dépasse les $50 \%$ et le record $(70 \%)$ revient à la petite commune de La Petite Fosse, voisine de Provenchères-sur-Fave. Globalement la rurbanisation y semble moins développée que sur le Plateau.

Carte 3 : La rurbanisation limitée des communes vosgiennes du Parc

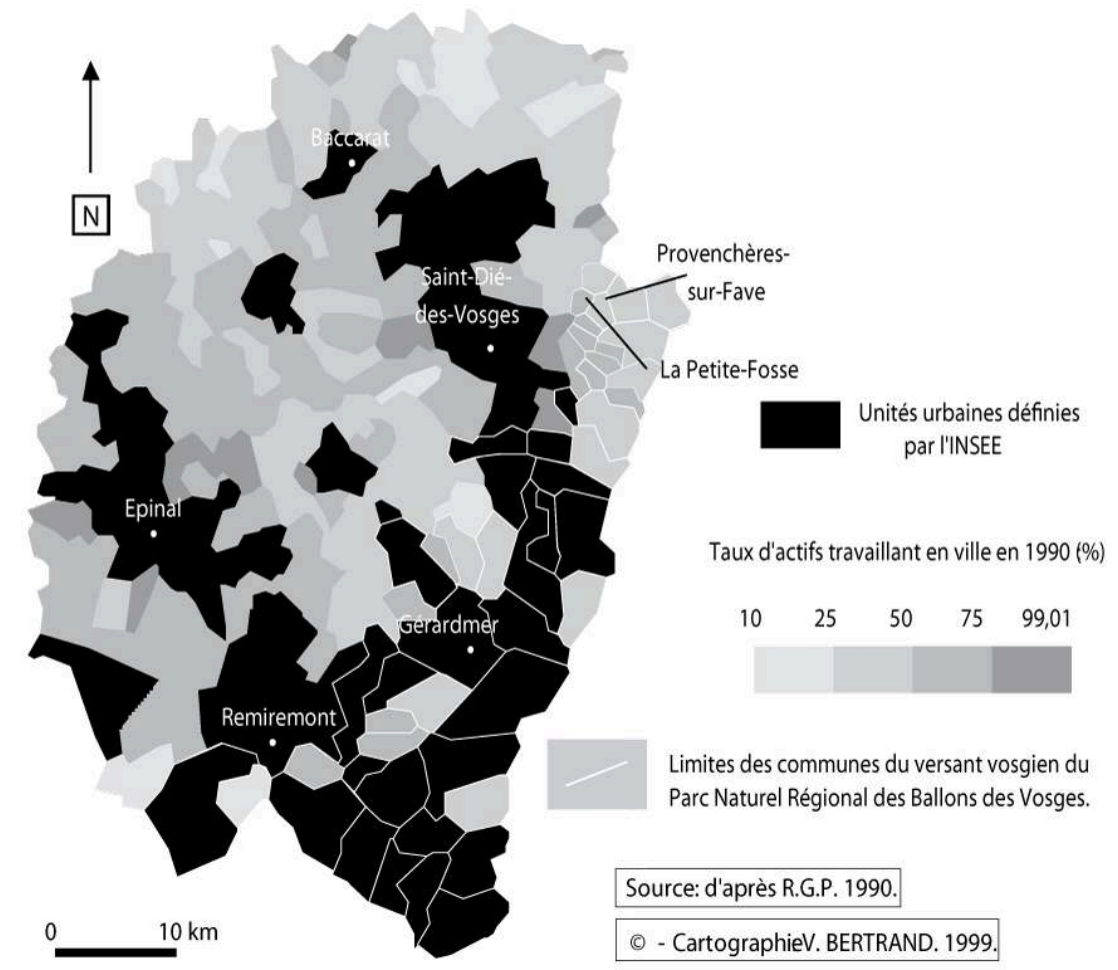

Aussi est-il nécessaire de recourir à une seconde approche consistant à mesurer l'attractivité de quelques villes. Les quatre exemples qui suivent (cartes $n^{\circ} 4$ à 7 ), sont particulièrement révélateurs du faible rôle des villes-portes ${ }^{2}$ et du rôle essentiel des petites villes du Parc. Epinal, préfecture de département, avec 35794 habitants en 1999 et un peu plus de 50000 habitants pour l'ensemble de son agglomération, est la plus peuplée, devant Saint-Dié-des-Vosges (avec une agglomération d'un peu moins de 30000 habitants) et Remiremont (un peu moins de 20000 ). 
Carte 4 : L'influence réduite et inégale d'Epinal sur les communes vosgiennes du Parc.

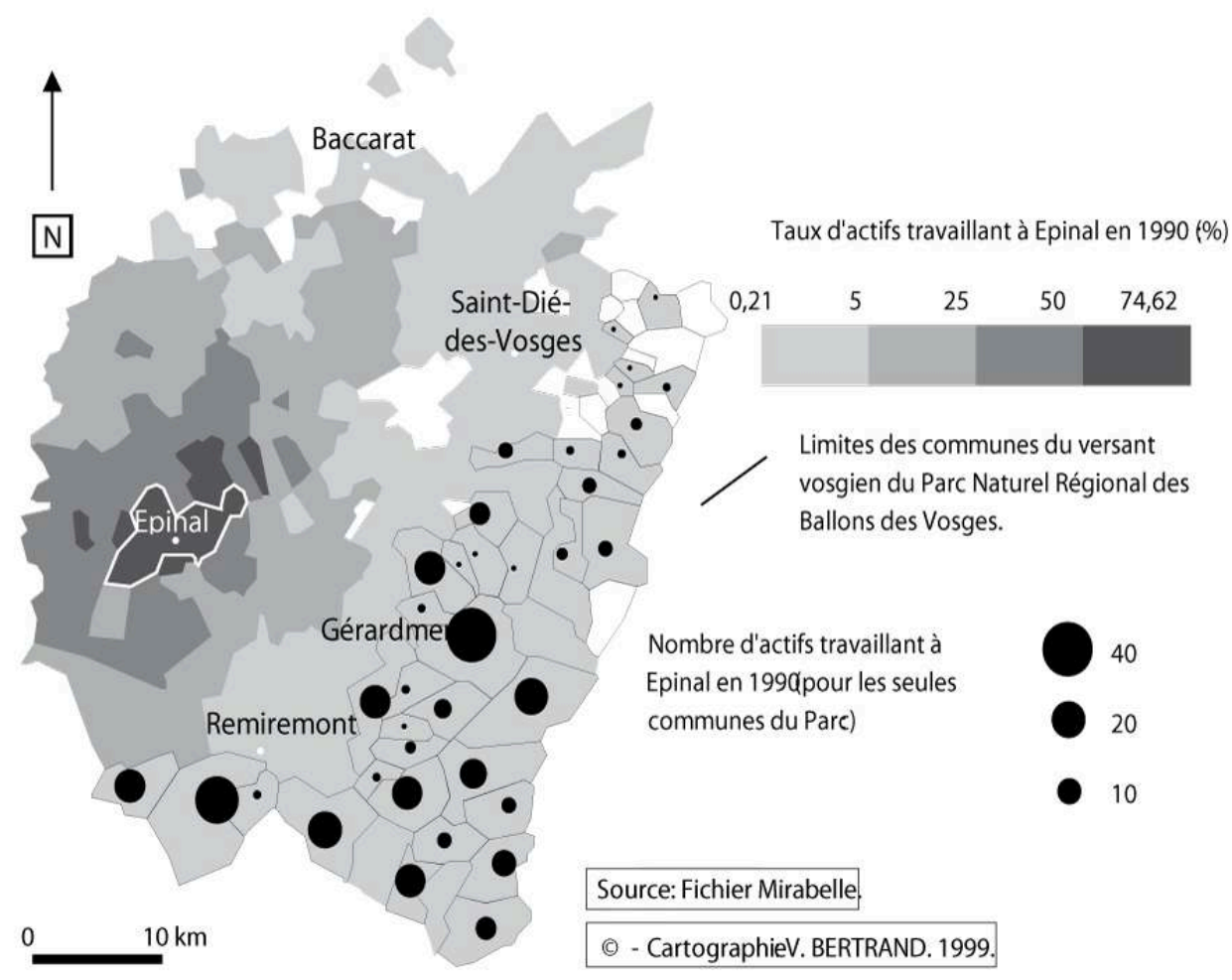

Carte 5 : La faible influence romarimontaine sur les communes vosgiennes du Parc.

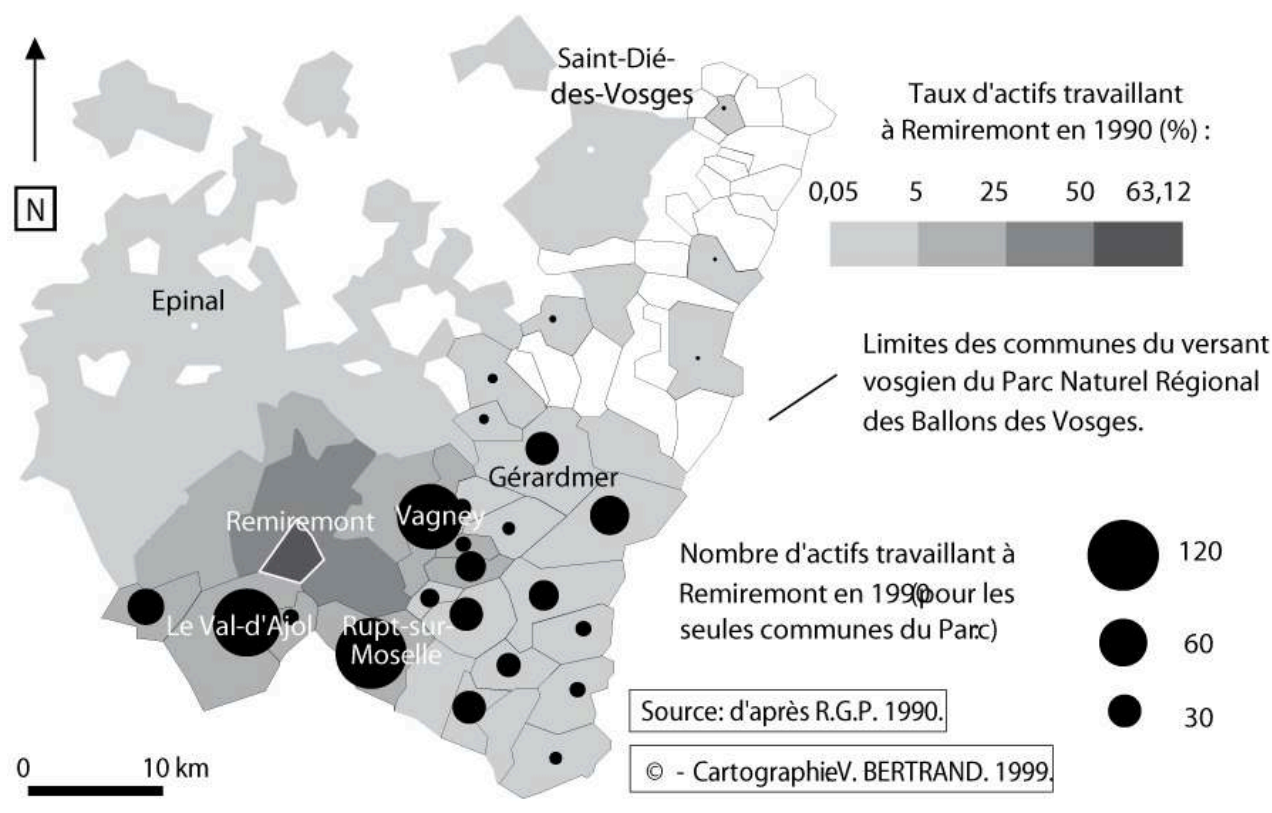


Carte 6 : L'influence déodatienne sur les communes vosgiennes du Parc.

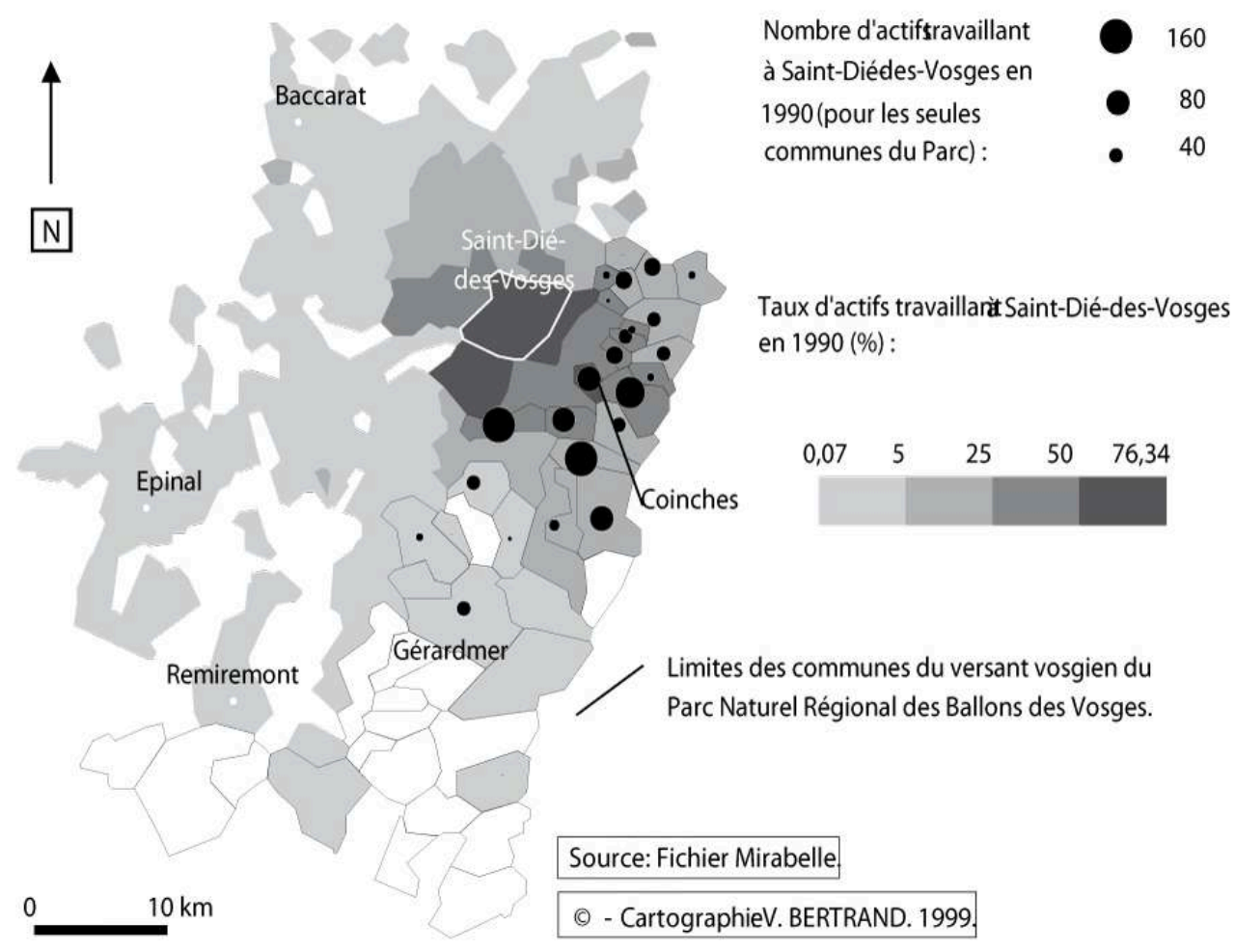

Carte 7 : Gérardmer : une influence limitée aux communes limitrophes.

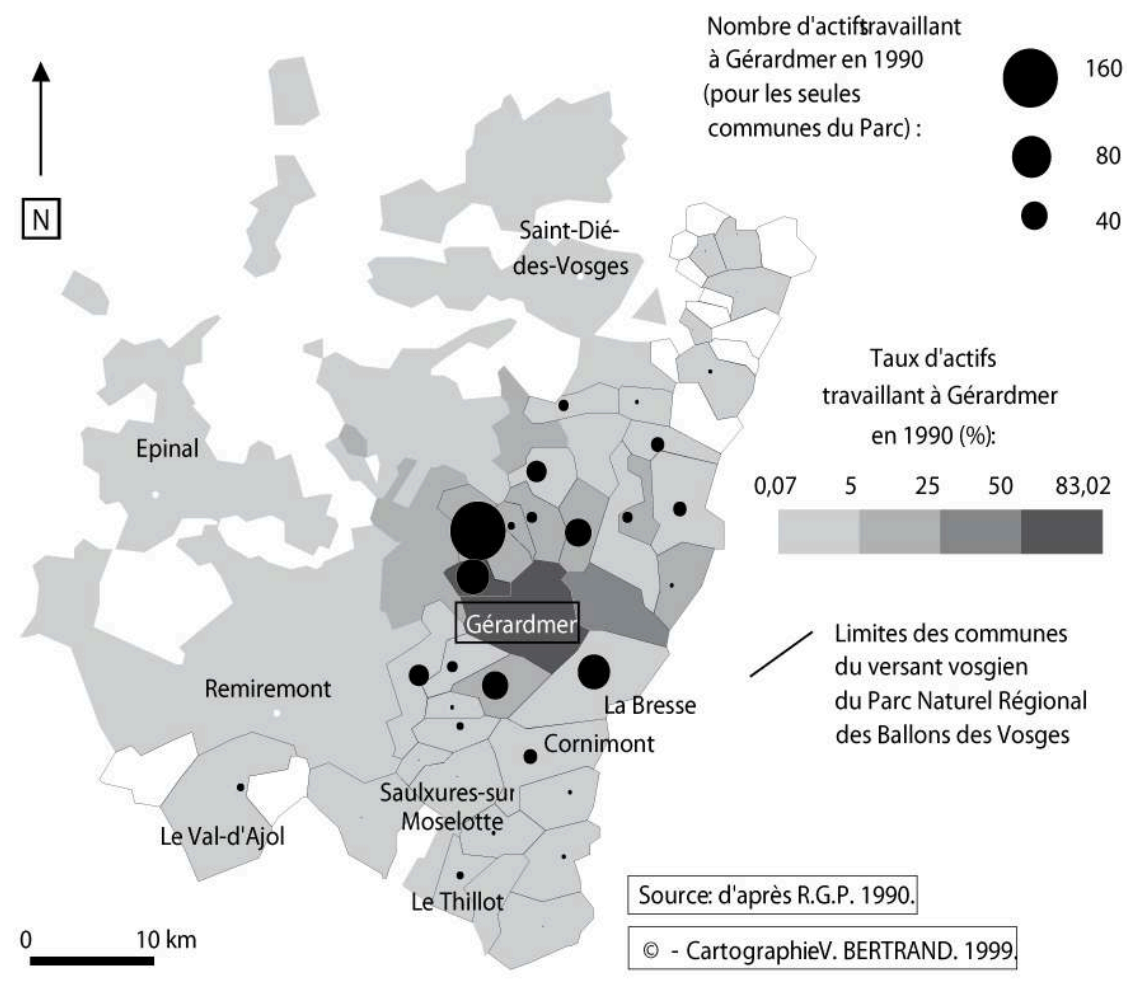

11 La carte $\mathrm{n}^{\circ} 4$ montre très nettement l'influence de la ville d'Epinal sur les communes les plus proches. Les communes du Parc, par contre, demeurent encore à l'écart de cette 
influence : Plombières-les-Bains, avec 2,7 \% d'actifs travaillant à Epinal est la commune du Parc qui subit la plus «forte influence » spinalienne. Et le flux le plus important de migrants pendulaires vers la Préfecture provient de Gérardmer: il compte 43 actifs. Incontestablement, en matière d'emploi, Epinal joue un rôle marginal dans la vie des communes vosgiennes du PNRBV. L'éloignement joue ici très certainement un rôle déterminant.

À l'inverse la situation de Remiremont, limitrophe du Parc, devrait-elle lui permettre d'exercer sur ces dernières une influence réelle. Il n'en est rien. En effet, la commune présentant le plus fort taux d'actifs travaillant à Remiremont est Rupt-sur-Moselle avec $8,7 \%$. On ne peut donc parler d'influence ou de dépendance entre ces deux communes. En fait, l'influence romarimontaine est circonscrite à quelques communes limitrophes. L'accessibilité joue ici un faible rôle. La faible taille de Remiremont, qui n'offre qu'environ 5000 emplois, (4 533 en 1999) et l'existence de nombreux emplois dans les communes de ce secteur du Parc explique la faiblesse de l'influence romarimontaine.

Saint-Dié-des-Vosges, par sa taille (22 569 hab.), du fait de la proximité du Parc et de la nature rurale de ce secteur préservé, exerce une certaine influence sur les communes du Parc les plus proches. Mais celle-ci se limite essentiellement à une petite dizaine de communes pour lesquelles le taux d'actifs travaillant à Saint-Dié-des-Vosges atteint 30 à $40 \%$. Coinches, petit village de 345 habitants $^{3}$ se démarque : $57 \%$ de ses actifs travaillant dans la sous-préfecture voisine. Pour le reste, les taux demeurent faibles. L'influence déodatienne se concentre dans un rayon d'une quinzaine de kilomètres et ne s'étend pas au sud de Gérardmer et La Bresse. Celles de Remiremont et d'Epinal se cantonnent au sud de ces deux villes. On retrouve l'opposition entre le nord et le sud de ce versant du Parc, autrement dit entre le bassin-versant amont de la Meurthe et celui de la Moselle.

Ainsi Saint-Dié-des-Vosges joue plus en matière d'emploi son rôle de ville-porte vis-àvis d'une partie du Parc que ne le font Remiremont et Epinal. Mais ce rôle demeure encore restreint. En fait le Parc est animé par une succession de petites villes dépassant rarement 5000 habitants, comme Cornimont, La Bresse, Saulxures-sur-Moselotte, Le Thillot, Le Val-d'Ajol ou Gérardmer. Cette dernière (carte $n^{\circ} 7$ ) illustre bien l'influence que joue ce genre de petites villes dans le Parc. Avec plus de 4000 emplois en 1990, Gérardmer peut satisfaire sa propre population active : 4000 actifs en 1990, (4 138 emplois en 1999 pour 3994 actifs) et ainsi attirer des actifs résidant dans les communes voisines. Cette attractivité parfois forte ( $45 \%$ des actifs de Xonrupt-Longemer travaillent à Gérardmer) se cantonne cependant aux communes limitrophes.

Ainsi la rurbanisation est assez difficile à appréhender à l'échelle du versant vosgien du Parc. Toutefois cette première approche montre une organisation de ce secteur du Parc relativement indépendante des villes-portes et davantage centrée sur les petites villes et les bourgs du Parc. Aussi est-il nécessaire de changer d'échelle pour mieux percevoir les divers aspects du phénomène.

\section{L'exemple de Provenchères-sur-Fave}

Petit chef-lieu de canton, Provenchères-sur-Fave compte 755 habitants en 1999, en légère hausse par rapport à 1990, et se situe à une dizaine de kilomètres à l'est de SaintDié-des-Vosges (carte $n^{\circ} 1$ ), dans le bassin de la Fave, affluent de rive droite de la 
Meurthe. Ce petit système villageois subit une mutation fonctionnelle profonde et progressive.

Sur le plan paysager, à l'instar de l'ensemble du Massif Vosgien la commune assiste depuis des décennies à la fermeture du paysage sous l'effet de la prolifération des bois et forêts sur les anciennes terres agricoles à l'abandon (carte $n^{\circ} 8$ ). Derrière ce bouleversement paysager se cache une profonde mutation des activités du village. Après avoir été dominé par des activités agricoles, sylvicoles et industrielles, le système devient tertiaire et dortoir.

Carte 8 : Provenchères-sur-Fave.

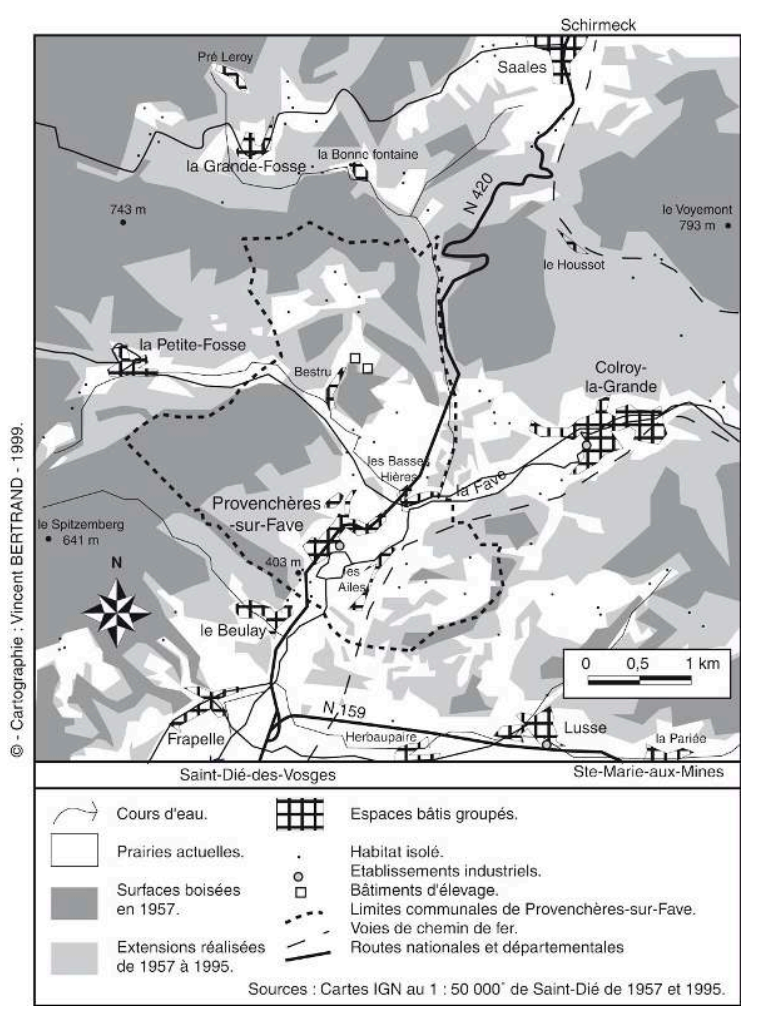

18 Les terres agricoles reculent et le nombre des exploitants est tombé à cinq: deux éleveurs de bovins, un éleveur de poulets en batterie, un éleveur de canards et une ferme-auberge subsistent. Pour survivre, la spécialisation ou la diversification sont ici quasi incontournables. En parallèle, l'activité industrielle, traditionnelle dans le Massif Vosgien, perdure grâce à une société d'étiquettes (ORALU) qui emploie une soixantaine de personnes, deux scieries et une fabrique de palettes. Le système est également pourvu en activités commerciales et en services. On y trouve une boucherie, une boulangerie, une pharmacie, un quincaillier, mais aussi une gendarmerie, une banque, un bureau de poste, une perception, un office notarial, un médecin, une école, et même un collège qui accueille environ 300 élèves originaires du canton mais aussi du canton voisin de Saales en Alsace.

19 Il est rare qu'une aussi petite commune puisse offrir autant de services à ses habitants. En fait Provenchères-sur-Fave doit ce privilège à son statut de chef-lieu de canton, mais aussi à sa nouvelle fonction résidentielle. De 1975 à 1990, le nombre des actifs travaillant en ville croît et leur poids passe de 26 à $40 \%$ de la population active communale. L'essentiel de ces migrants pendulaires se rend à Saint-Dié-des-Vosges et 
dans sa banlieue. En parallèle, le nombre des actifs travaillant sur place décline et celui des actifs sortant vers les villages voisins s'effondre. Le poids grandissant des rurbains dans le village concourt aussi à la rentabilité et au maintien des commerces et services du système. Aussi le phénomène profite-t-il ici au village mais aussi à tout le canton. Sans ces rurbains, certains commerces et services auraient disparu. Le système présente à la fois les traits d'un petit bourg rural assurant les services de proximité pour son canton et ceux d'une commune-dortoir rurbaine et de plus en plus dépendante du pôle voisin. Ces deux fonctions illustrent les liens qui unissent les villages à un bourg et ce dernier à un pôle. Elles sont complémentaires et garantes de la pérennité du système. Toutefois, pour agir face à une telle organisation de l'espace, qui dépasse largement le territoire communal, il importe de disposer de structures administratives adaptées. Le canton s'est structuré au profit d'une communauté de communes, celle de la Fave, qui rassemble les villages du canton, mais certaines compétences lui échappent pour des raisons de territorialité. De fait, plusieurs SIVU aux limites différentes coexistent. L'un a la charge du ramassage scolaire, un autre celle de la gendarmerie, un troisième celle du câblage télévisuel. À ces structures s'ajoute le PNRBV qui travaille, entre autres, à la réouverture des paysages.

Enfin depuis la loi Voynet, les Pays se multiplient et la Déodatie émerge rapidement. Si l'apparition de structures et de territoires nouveaux peut être l'occasion d'appréhender des phénomènes comme la rurbanisation, il n'en demeure pas moins sûr que la superposition des structures risque de faire perdre énergies et ressources financières si elles ne définissent pas avec précision leurs zones d'intervention respectives. Le système a su trouver un certain équilibre en se jouant des échelles et en multipliant les fonctions. L'organisation administrative du territoire saura-t-elle en faire autant ?

\section{BIBLIOGRAPHIE}

BAUER G. et ROUX J.M. (1976). - La rurbanisation ou la ville éparpillée, coll. Espacements, Paris, Seuil, $190 \mathrm{p}$.

BERGER M. (1992). - La périurbanisation dans les pays développés d'économie libérale : repères bibliographiques, Villes en parallèle, ${ }^{\circ} 19$, p. 47-62.

BERGER M., FRUIT J.P., PLET F., ROBIC M.C. (1980). - Rurbanisation et analyse des espaces ruraux périurbains, L’Espace Géographique, T.IX, ${ }^{\circ}$ 4, , p. 303-313.

BERTRAND V. (1998). - Quand villes et campagnes se confondent, Économie Lorraine, n 179, p. 11-14.

BERTRAND V. (1999). - Les espaces rurbains en Lorraine: du paysage au système géographique. L'exemple du Lunévillois, Thèse, Université Nancy2, 423 p.

BÉTEILle R. et MontAGNÉ-VILLETTE S. (dir.) (1995). - Le « rural profond » français, DIEM, nº 18, Paris, SEDES, $166 \mathrm{p}$.

Bonnefont J.C. et al. (1996). - Lorraine, Le Puy-en-Velay, Christine Bonneton éd., 1984, 417 p. 
CCI de Saint-Dié-des-Vosges et d'Epinal : Le tissu industriel des Vosges, 27 p.

DAT Conseil (1998). - Etude sur l'organisation et le fonctionnement des territoires du Massif Vosgien, (rapport pour l'Association du Massif Vosgien).

DÉZERT B., METTON A., STEINBERG J. (1991). - La périurbanisation en France, Paris, CDU-SEDES, 226 p.

FRÉCAUT R. (dir.) (1983). - Géographie de la Lorraine, Nancy, PUN-Serpenoise, 636 p.

INSEE Lorraine (1997). - Zones d'emploi de Remiremont - Gérardmer, Economie Lorraine, $\mathrm{n}^{\circ}$ 160, janvier.

INSEE Lorraine (1998). - Zone d'emploi de Saint-Dié-des-Vosges, Economie Lorraine, $\mathrm{n}^{\circ} 178$, septembre.

KAYSER B. (1990). - La renaissance rurale. Sociologie des campagnes du monde occidental, Paris, A.

Colin, $316 \mathrm{p}$.

LEROUX P., MADELLA A., TACQUARD F. et G. (1997). - La Lorraine et ses paysages, Metz, Agence Régionale de l'Environnement en Lorraine (AREL) et Direction Régionale de l'Environnement (DRE), $24 \mathrm{p}$.

MONNOT S. et TRAYNARD É. (1999). - Recensement de la population lorraine, Économie Lorraine Résultats, Nancy, INSEE, $\mathrm{n}^{\circ} 11$, juillet, $4 \mathrm{p}$.

NonN H. (1999). - Recomposition socio-économique et territoriales contemporaine dans le Massif vosgien, in coll. (J.P. Husson dir.), Territoires, paysages et héritages, PUN.

\section{Sources statistiques}

INSEE : R.G.P. 1990 et 1999, fichier Mirabelle 1990.

\section{RÉSUMÉS}

La rurbanisation est un phénomène récent et particulièrement dynamique des pays développés. Après en avoir donné une définition originale, nous envisageons son étude selon deux échelles : l'une reprenant l'aire d'influence des villes, l'autre correspondant à l'échelon communal. Ces deux échelles impliquent l'usage de démarches complémentaires : la première, quantitative, s'attache aux flux pendulaires. La seconde, paysagère, concerne l'organisation systémique du village. Nous limiterons notre étude au versant vosgien du Parc Naturel Régional des Ballons des Vosges.

Rurbanisation is a recent and especially dynamic phenomenon in developed countries. Following an initial definition, we study it at two scales: one covering the area of influence of towns, the other corresponding to village level. Both imply the use of complementary approaches: the first, quantitative, is concerned with commuters; the second uses landscape to understand village organisation. We limit our study to the Vosgian side of the Ballons des Vosges Natural Regional Park.

Die Wanderung im Stadtumland ist ein rezenter und dynamischer Prozess in den Industrieländern. Wir schlagen eine originale Definition der Stadtumlandwanderung vor und führen eine Untersuchung auf zwei Ebenen durch: auf den zentralörtlichen Einzugsbereichen und auf der Ebene der Gemeinde. Diese beiden Ebenen führen zu zwei sich ergänzenden Wegen: Der erste quantitative Weg untersucht die Pendlerströme, der zweite Weg, der sich an die Landschaft interessiert, betrifft den Aufbau des Dorfes. Wir begrenzen unsere Untersuchung auf den Vogesenteil des regionalen Naturparks der Ballons der Vogesen. 
INDEX

Schlüsselwörter : Landschaften, Pendelwanderer, Regionaler Naturpark der Ballons in den Vogesen, rurbanisation

Mots-clés : migrants pendulaires, Parc Naturel Régional des Ballons des Vosges, paysages, rurbanisation

Keywords : Ballons des Vosges Natural Regional Park, commuters, landscape, rurbanisation

\section{AUTEUR}

\section{VINCENT BERTRAND}

CERPA / Université de Nancy 2 - BP 33-97 - 54015 NANCY cedex - vincent.bertrand@univnancy2.fr 